\title{
Beta-haemolytic streptococci in saliva*
}

\author{
By P. W. ROSS \\ Department of Bacteriology, Medical School, University of Edinburgh
}

(Received 21 December 1970)

\begin{abstract}
SUMMARY
Viable counts of beta-haemolytic streptococci per ml. of saliva were made in the following groups: (1) children with acute streptococcal sore throat, (2) children with acute non-streptococcal sore throat, (3) children who had no sore throat but were streptococcal throat carriers, (4) children who neither had a sore throat nor were streptococcal throat carriers.

The mean counts from cases of streptococcal sore throat and from streptococcal carriers were respectively $1.4 \times 10^{6}$ and $2.5 \times 10^{5}$ per $\mathrm{ml}$.

In a comparison of the efficiency of the throat swab, sublingual swab and specimen of saliva in isolating beta-haemolytic streptococci from the upper respiratory tract, culture of saliva produced the best results.
\end{abstract}

\section{INTRODUCTION}

The literature contains comparatively few references to the presence of betahaemolytic streptococci in the saliva. Hare (1940) reported their presence, as did Hamburger (1944), who studied the incidence of beta-haemolytic streptococci in the saliva of army recruits who were suffering from scarlet fever, pharyngitis or tonsillitis. He isolated these organisms from $64 \cdot 6 \%$ of 527 cultures of saliva, and found that the numbers of organisms ranged from $10^{2}$ to $5 \times 10^{6}$ per ml. of saliva; the majority, however, fell within the range of $10^{3}$ to $10^{6}$ per ml. Hamburger also stated that patients with heavily positive throat cultures could yield negative salivary cultures and that those with scanty growth from the throat could have large numbers of beta-haemolytic streptococci in the saliva. Duguid (1946a) swabbed 87 people who had beta-haemolytic streptococci in the throat, 50 of whom had scarlet fever and 37 of whom were streptococcal carriers, and isolated these organisms, usually in small numbers, from the mouth secretions of 13. Hamburger \& Robertson (1948) reported a very high carrier rate in convalescent and healthy carriers, the average count being in the region of $8.0 \times 10^{5}$ per ml. of saliva. Rubbo \& Benjamin (1953) examined saliva from nine streptococcal carriers and reported a wide range of counts. The degree of growth obtained from the throat swabs bore no relation to the concentration of beta-haemolytic streptococci in the saliva.

Holmes \& Williams (1958) investigated anterior mouth swabs from children in

\footnotetext{
* Based on part of a thesis accepted for the degree of M.D., University of Aberdeen.
} 
Dr Barnardo's homes and isolated beta-haemolytic streptococci from $62 \%$ of 78 children who had these organisms in their throats. In their studies on crossinfection in isolation hospitals Boissard \& Fry (1966) reported that beta-haemolytic streptococci could be isolated from the mouths of almost half the patients who had scarlet fever and concluded that the presence of these organisms in the saliva was a feature of the early stages of tonsillar infection. They recommended the use of a nasal and a salivary swab rather than a nasal and throat swab to detect dispersers.

In this study quantitative counts of beta-haemolytic streptococci from the saliva of four groups of children were performed:

(1) Children with streptococcal sore throat.

(2) Children with non-streptococcal sore throat.

(3) Children who had no sore throat but were throat carriers of beta-haemolytic streptococci.

(4) Children who neither had a sore throat nor were throat carriers of betahaemolytic streptococci.

The relative merits of throat swabs, sublingual swabs and salivary culture in isolating beta-haemolytic streptococci from the throat were evaluated.

\section{Selection of children}

\section{MATERIALS AND METHODS}

In the investigations of children with sore throat the family doctors obtained the specimens of saliva when they examined the children. Throat swabs were also taken at that time and the cases were divided into those from whom beta-haemolytic streptococci had been isolated and those from whom these organisms had not been isolated. The streptococcal sore-throat group comprised 47 children and the non-streptococcal sore throat group 52. Children who had no sore throat but were streptococcal throat carriers, and the control group (those who had no sore throat and were not streptococcal throat carriers), were selected from schools in the PiltonMuirhouse areas in Edinburgh. There were 60 children in each of these two groups, and these were selected for the study whenever the bacteriological results of routine weekly swabbings at these schools became available. The control group was matched as closely as possible with the three other groups in age, sex, season and family size.

A completely separate group of children was investigated in evaluating the throat swab, sublingual swab and salivary culture in the isolation of beta-haemolytic streptococci from the upper respiratory tract. This group comprised 248 children who were found to be symptomless streptococcal throat carriers at routine weekly throat swabbing sessions in schools. No salivary examinations were performed on them at these routine visits; they were included in the study group only because throat-swab culture had produced positive results. These children were revisited at school one or two days after their first throat swab had grown beta-haemolytic streptococci and a further throat swab, as well as a sublingual swab and specimen of saliva, were obtained at this time. 


\section{Specimen collection}

Specimens of saliva from the acute cases were obtained by the family doctors and from the carriers and controls by the author. A specially prepared kit was used, which consisted of a sterile Pasteur pipette calibrated to deliver $0.02 \mathrm{ml}$. drops contained in a sterile test-tube. This test-tube had a metal cap, and cotton-wool at the foot to prevent damage to the pipette. The pipette was placed on the floor of the anterior mouth and saliva sucked up by means of a rubber teat. Occasionally it was difficult to get saliva from children, especially from those who were apprehensive. No sialogogues were used but, on the promise of a sweet, saliva was obtained from most of the previously recalcitrant children. The 248 children were swabbed twice, but apart from this group no child was sampled on more than one occasion and a specimen of saliva was taken from each child once only. As well as specimens of saliva, throat and sublingual swabs were taken. 'Exogen' swabs, which had tips of buffered absorbent cotton-wool sterilized by ethylene oxide, were used, since beta-haemolytic streptococci had been found to remain viable on these for up to $24 \mathrm{hr}$. (Ross, 1970).

\section{Laboratory methods}

Saliva and swabs were processed within $2 \mathrm{hr}$. of being obtained. Estimation of the number of beta-haemolytic streptococci was made by surface-viable counts (Miles \& Misra, 1938). Saliva was diluted 1/4 with distilled water and tenfold serial dilutions were made from this. Blood agar plates incubated aerobically and crystal violet blood agar plates incubated anaerobically for 14-18 hr. were used for the streptococcal counts.

Sublingual and throat swabs were plated out on blood agar and crystal violet blood agar and incubated as above. Grouping of the beta-haemolytic streptococci was by the acid extraction method (Lancefield, 1933) and by the use of a bacitracin disk (Maxted, 1953) ; groups A, B, C and G were tested for. Strains were screened by the bacitracin disk method and if they were sensitive to bacitracin they were taken to be group A; no serological confirmation was obtained. This occurred at a later stage when presumptive group A cultures were typed; if any were untypable they were then tested to ensure that they did in fact belong to group A. All bacitracinresistant strains, however, were grouped by Lancefield's method. Typing was by slide agglutination (Griffith, 1934) using T-antisera (Williams, 1958).

\section{RESULTS}

All 47 children with streptococcal sore throat had beta-haemolytic streptococci in the saliva. Mean counts per ml. of saliva varied from child to child and the range was from $4.8 \times 10^{5}$ to $4.4 \times 10^{6}$, with a mean of approximately $1.4 \times 10^{6}$. The distribution of these counts is seen in Table 1. This table also shows the distribution of counts obtained from the symptomless streptococcal throat carriers. Fifty-five out of sixty $(91.7 \%)$ had beta-haemolytic streptococci in the saliva, but mean counts 
per ml. were considerably lower than those obtained from the acute cases. They ranged from $4 \times 10^{3}$ to $3.2 \times 10^{6}$, with a mean of approximately $2.5 \times 10^{5}$ per ml.

Of the 102 pairs of beta-haemolytic streptococci isolated from throat and saliva of the cases of streptococcal sore throat and also the symptomless throat carriers, 99 pairs $(97 \%$ ) belonged to group A; 46 from the 47 cases and 53 from the 55 symptomless carriers produced growth of these organisms. Two of the non-group A pairs belonged to group $G$, but the other was ungroupable. The streptococcal group in the throat was the same as that in the saliva in each child.

Table 1. Distribution of salivary counts of beta-haemolytic streptococci in 47 cases of acute streptococcal sore throat and in 55 streptococcal throat carriers

$\begin{array}{ccc}\begin{array}{c}\text { Salivary count } \\ \text { (millions } / \mathrm{ml} \text {.) }\end{array} & \text { Cases } & \text { Carriers } \\ <0 \cdot 1 & 0 & 31 \\ 0 \cdot 1-0 \cdot 49 & 5 & 19 \\ 0 \cdot 5-0 \cdot 99 & 15 & 3 \\ 1 \cdot 0-1 \cdot 49 & 13 & 1 \\ 1 \cdot 5-1 \cdot 99 & 2 & 0 \\ 2 \cdot 0-2 \cdot 49 & 5 & 0 \\ 2 \cdot 5-2 \cdot 99 & 1 & 0 \\ 3 \cdot 0-3 \cdot 49 & 1 & 1 \\ 3 \cdot 5-3 \cdot 99 & 3 & 0 \\ 4 \cdot 0-4 \cdot 49 & 2 & 0\end{array}$

The group A organisms from the cases and carriers were typed; in two of the carriers the organisms from both the throat and saliva were untypable, but the remaining 97 Strep. pyogenes from both throat and saliva were typed. Eighty-seven $(89 \%)$ of the strains in the throat and saliva were of the same serotype; two cases and eight carriers had different serotypes.

Fifty-two children had sore throats from which no beta-haemolytic streptococci had been isolated, but three had these organisms in the saliva, at counts of $10^{5}$; $2.4 \times 10^{5}$ and $9 \cdot 6 \times 10^{5}$ per ml. One child had group A organisms and the others group G. Three of the 60 control children, who had no sore throat and from whose throats no beta-haemolytic streptococci had been isolated, had these organisms in the saliva; the counts were $2.4 \times 10^{5} ; 3 \times 10^{5}$ and $3.8 \times 10^{5}$ per ml. One had group A, another group $\mathrm{C}$ and the third ungroupable organisms.

Beta-haemolytic streptococci were grouped using antisera to groups A, B, C and G; 210 strains were grouped, comprising 47 pairs from the throat and saliva of the acute streptococcal cases; 55 pairs from both throat and saliva of the symptomless carriers; 3 strains from the saliva of the non-streptococcal cases of sore throat; and 3 strains from the saliva of the controls. Two hundred (95\%) belonged to group A and $6(2.9 \%)$ to group $\mathrm{G}$; only 1 belonged to group $\mathrm{C} ; 3$ were ungroupable. The bacitracin screening test correctly identified $99 \%$ of group A and $88.9 \%$ of nongroup-A strains.

Table 2 shows the growths obtained from culture of throat swabs, sublingual swabs and salivary specimens from the separate group of 248 school-children with 
symptomless streptococcal throat carriage, and it can be seen that whereas previous throat-swabbing had produced growth of beta-haemolytic streptococci from each child, repeat throat-swabbing produced growth from only $66.9 \%$.

$\begin{array}{lrc}\text { Table 2. Growth of beta-haemolytic streptococci from the throat swab, } \\ \text { sublingual swab and specimen of saliva from } 248 \text { school-children } \\ & \text { No. } & \% \\ \text { Growth from throat swab } & 166 & 66 \cdot 9 \\ \text { Growth from sublingual swab } & 56 & 22 \cdot 6 \\ \text { Growth from saliva } & 236 & 95 \cdot 1\end{array}$

\section{DISCUSSION}

Beta-haemolytic streptococci were isolated from the saliva of 102 out of 107 $(95 \cdot 3 \%)$ streptococcal sore throat cases and symptomless carriers. This isolation rate compares with that reported by Hamburger \& Robertson (1948) but is higher than those reported by Hamburger (1944), Duguid (1946a) and Rubbo \& Benjamin (1953).

Although the mean count in the cases of sore throat, $1.4 \times 10^{6}$ per ml., differed considerably from the $2.5 \times 10^{5}$ per ml. found in the carriers, it is impossible to draw a line of demarcation between counts obtained from cases and carriers. In general, however, those of the sore throat cases were above $5 \times 10^{5}$ per $\mathrm{ml}$., although cases with low counts occurred, and those of the carriers were below this figure. Important findings were that not only in $97 \%$ of children were group A organisms present in both throat and saliva, but that in $89 \%$ the group A serotypes in both situations were identical. Clearly the saliva is a reliable indicator of the presence of beta-haemolytic streptococci in the throat in the majority of cases.

Isolation of streptococci of one group from the throat and of another group from the saliva did not occur in any of the cases or carriers, but in ten children the group A serotypes isolated from the sore throat and saliva were different. This latter finding does not necessarily mean that quite separate serotypes colonized both sites, because it is possible that the colonies of beta-haemolytic streptococci which were typed randomly did not represent the predominant serotype of either site.

The isolation rate of Strep. pyogenes was high and that of the other groups very low. It was interesting to note scarcely any difference between the cases of streptococcal sore throat and the symptomless throat carriers in the isolation rate of Strep. pyogenes. This could not be explained by the presence of a particularly virulent serotype in the community because the T-agglutination patterns of the group $A$ strains were very varied. The number of ungroupable strains of beta-haemolytic streptococci was insignificant; strains were ungroupable presumably because they belonged to groups other than A, B, C or G.

The bacitracin disk tests proved satisfactory and results correlated well with serological grouping of the strains. The grouping regime used in the present series can be employed with accuracy only if typing is also performed. Because there is a slightly greater chance of group A strains being resistant than non-group A strains 
sensitive to bacitracin (Maxted, 1953), and because group A strains are generally far more frequent than non-group A strains in the community, it is economical in time and materials to confine serological grouping initially to bacitracin-resistant strains. There is no need to group bacitracin-sensitive strains serologically if typing is to be performed, because if any strain proves untypable its group can then be checked serologically. Two such strains were detected in the present series.

With regard to the second part of this study, it can be seen in Table 2 that culture of saliva appears to be the most efficient way of recovering beta-haemolytic streptococci from the upper respiratory tract. An isolation rate of $95.1 \%$ is very high and that of $66.9 \%$ from the throat swab surprisingly low. The isolation rate using the sublingual swab $(22.6 \%)$ is very low and the discrepancy between the results of this and culture of saliva is considerable. Growth from all three specimens occurred simultaneously in only $17.7 \%$ of the children. Cases or carriers not detected by the sublingual swab were mostly those with very few organisms in the saliva. Duguid (1946a) isolated beta-haemolytic streptococci from the saliva of only 13 out of 87 persons who had these organisms in the throat and this poor isolation rate may well have been due to his use of sublingual swabs.

These results clearly demonstrate that saliva from certain cases of sore throat may contain many beta-haemolytic streptococci and that saliva from others may contain few. Duguid (1946b) stated that in the production of true airborne infection by droplet spray the content of the saliva was important. He added, however, that the danger was appreciable only when the saliva was heavily infected. These studies show that the saliva can indeed be heavily infected and it may be that these people should be included in the category of 'dangerous carriers' which Hamburger, Green \& Hamburger (1945) reserved for those who were nasal carriers of betahaemolytic streptococci. An important investigation would be to find out which of the following categories is the most important from the point of view of spreading infection: nasal carriage, nasal and salivary carriage, and salivary carriage.

Clearly culture of the saliva is very useful, and combined with the use of a throat swab will provide maximum numbers of isolations of beta-haemolytic streptococci from cases of sore throat. The sublingual swab is not considered to be of value in studies on streptococcal sore throat.

I am indebted to Dr M. T. Parker and Dr W. R. Maxted for providing antisera and for giving invaluable advice on matters pertaining to streptococci. I am deeply grateful to them and to the staff of the Streptococcus Reference Laboratory, Colindale, for solving difficult problems in the typing of the streptococci.

I also acknowledge with thanks the co-operation and assistance of Drs E. V. Kuenssberg, J. D. E. Knox, G. MacNaughtan, A. R. Laurence and A. A. Robertson. 


\section{REFERENCES}

BoIsSARD, J. M. \& FRY, R. M. (1966). Streptococcal school outbreaks: a method of investigation and control. Journal of Hygiene 64, 221.

DUGUID, J. P. (1946a). Expulsion of pathogenic organisms from the respiratory tract. British Medical Journal i, 265.

DUGUID, J. P. (1946b). The size and duration of air carriage of respiratory droplets and droplet nuclei. Journal of Hygiene 44, 471.

GRIFFITH, F. (1934). The serological classification of Streptococcus pyogenes. Journal of Hygiene 34, 542 .

HAMBURGer, M., JR. (1944). Studies on the transmission of hemolytic streptococcus infections. II. Beta-hemolytic streptocci in the saliva of persons with positive throat cultures. Journal of Infectious Diseases 75, 71.

Hamburger, M., Jr., Green, M. J. \& Hamburger, V. G. (1945). The problem of the 'dangerous carrier' of hemolytic streptococci. I. Number of hemolytic streptococci expelled by carriers with positive and negative throat cultures. Journal of Infectious Diseases 77, 68.

Hamburger, M., JR. \& Robertson, O. H. (1948). Expulsion of group A hemolytic streptococci in droplets and droplet nuclei by sneezing, coughing and talking. American Journal of Medicine 4, 690.

HARE, R. (1940). The expulsion of hemolytic streptococci by nasopharyngeal carriers. Canadian Public Health Journal 31, 539

Holmes, M. C. \& Williams, R. E. O. (1958). Streptococcal infections among children in a residential home. I. Introduction and definitions: the incidence of infection. Journal of Hygiene 56, 43.

LANCEFIELD, R. C. (1933). A serological differentiation of human and other groups of hemolytic streptococci. Journal of Experimental Medicine 57, 571.

MAXTED, W. R. (1953). The use of bacitracin for identifying group A haemolytic streptococci. Journal of Clinical Pathology 6, 224.

Mires, A. A. \& MisRA, S. S. (1938). The estimation of the bactericidal power of the blood. Journal of Hygiene 38, 732.

Ross, P. W. (1970). M.D. thesis, University of Aberdeen.

Rubbo, S. D. \& Benjamin, M. (1953). Transmission of haemolytic streptococci. Journal of Hygiene 51, 278.

WinImams, R. E. O. (1958). Laboratory diagnosis of streptococcal infections. Bulletin of the World Health Organization 19, 153. 